\title{
Our new journal: JA Clinical Reports
}



As announced in this issue's editorial titled "Launch of $J A$ Clinical Reports: Anesthesia crisis in Japan" by Dr. Kazuyoshi Hirota, we have decided to establish a new journal, $J A$ Clinical Reports. We hope that it will lead to an increase in the number of publications of high-quality clinical research and will encourage junior anesthesiologists. JA Clinical Reports is an open access, peer-reviewed online journal and will consider any original case reports in anesthesiology.

On behalf of the Japanese Society of Anesthesiologists, we eagerly look forward to your submissions.
Journal title: JA Clinical Reports

Publishing format: electronic online edition only, open access

Frequency of publication: continuous publishing (1 volume per year)

Contents: Case Reports, Clinical Research Letters (Short Communications), Letters to the Editor Initial publication: November 2015

Article processing charge: no charge for members of the Japanese Society of Anesthesiologists; $€ 1250$ for non-members

Submission guidance: use the online system Editorial Manager starting June 2015

For detailed instructions for submissions, please see the instruction for authors of JA Clinical Reports.

Inquiries:

Japanese Society of Anesthesiologists

Kobe Kimek Center Building 3F

1-5-2, Minato-Minami machi, Chuo-ku

Kobe 650-0047, Japan

E-mail: gakujyutu@anesth.or.jp

JA Clinical Reports

Editor-in-Chief

Mchiaki Yamakage, MD, PhD 